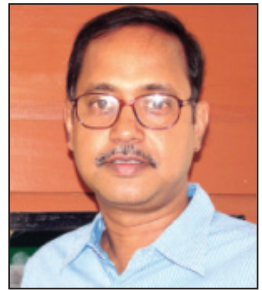

JOURNAL OF MINES, METALS \& FUELS www.jmmf.info

DISCUSSION-OPINION-EDITORIAL

Jayanta Bhattacharya

Hony. Chief Editor

\title{
How to induct and adopt automation in the minerals, oil and gas sector
}

An explanation of the term industrial automation can be the creation and application of technology to monitor and control the production, and inbound and outbound logistics for the delivery of products and services to the customers. As on date automation can be divided into two groups: robotic process automation and intelligent process automation. Both these two groups are evaluated in terms of engineering applications, system design and business review. With the recent developments in robotic process automation (RPA) and artificial intelligence (AI), academics and industrial practitioners are now pursuing robust and adaptive decision making (DM) in real-life engineering applications and automated business workflows and processes to accommodate context awareness, adaptation to environment and customization.

At present there are three areas of automation thinking in the mining industry and they are:

I. Automation.

II. Digitalization.

III. Digitally enabled business and strategy implementation.

In the area of automation the current focus is on artificial intelligence, algorithm and program design, electronic control technology, programmable logic controller, robotics and mechatronics

Unlike digitization, which is a mere technical phenomenon, digitalization has impacts on society and the economy through fostering, for example, communication, mobility, speed, virtualization, dissolution of boundaries, interconnectedness, market transparency, and competition. Hence, we use the term "digitalization" to refer to the socio-technical change invoked by digital technologies and their confluence in digital infrastructures.

Both automation and digitalization are largely confined to production and surrounding sites. The third area, digitally enabled business and strategy implementation will prepare the companies to face increasing market risk with fast assessment - response feedback; collaborative judgments on decision will be implemented with balance and agility. Today's online retail giants are the case examples. These are mostly beyond mines where the produced minerals are primed for business across different clients and customers. The digital platforms will be the backbone for conducting strategic planning - beyond operational and production planning. 